\title{
Neural lineage development of rhesus monkey embryonic stem cells: Insight of neurogenesis and gliogenesis in vitro
}

Hongwei Chen ${ }^{1,5}$, Yu Mao ${ }^{2,5}$, Bin $\mathrm{Li}^{1,5}$, Qiang Wei ${ }^{1,5}$, Jing Zhang ${ }^{1,5}$, Jinhuan Wang ${ }^{4}$, Jianhong Wang ${ }^{2,5}$, Shufen Wang $^{1,5}$, Tao Tan ${ }^{1,5}$, Xiuzhen Zhang ${ }^{1,5}$, Jian $\mathrm{Li}^{3}$, Yuanye $\mathrm{Ma}^{2}$, Weizhi $\mathrm{Ji}^{1}$

${ }^{1}$ Laboratory of Reproductive and Developmental Biology, Kunming Primate Research Center and Kunming Institute of Zoology, CAS; ${ }^{2}$ Laboratory of Primate Recognition Neuroscience, Kunming Primate Research Center and Kunming Institute of Zoology, CAS, Kunming Yunnan, China; ${ }^{3}$ Central Laboratory, ${ }^{4}$ Key Laboratory of Cellular and Molecular Evolution, Kunming Institute of Zoology, CAS,Kunming Yunnan, China, ${ }^{5}$ Graduate School of Chinese Academy of Sciences, Beijing, China

Neurons and glia are important targets of embryonic stem (ES) cell research, promising a renewable source of these differentiated cells for basic research and regenerative medicine. Though many subtypes of nerve cells have differentiated from ES cells in vitro, their lineal relations are not clear. In the present study, early neurogenesis and gliogenesis of rhesus monkey ES cells (rESCs) was investigated in vitro. Our findings revealed that the predominant process of neural differentiation progressed with a sequential lineage restriction from neuroepithelial stem/ progenitor (NEP) cells to neurons and glia. Temporally, the majority of NEP cells switched into radial glia (RG) dynamically, and then developed into neurons and glia via neuronal restricted precursors (NRPs) and glial restricted precursors (GRPs), respectively. Immunostaining and RT-PCR also demonstrated that neural progenitors derived from ES cells displayed spatial identity of both dorsoventral and anteroposterior axes of the early neural tube, with posterior codes enhanced within subsequent passages. Further pharmacological assay implied that Notch signaling regulated the maintenance of the identity of the neural stem/progenitor cells, as well as neuronal fate determination. FGF via specific receptors, and Notch signaling pathways might cooperate to manipulate cell survival, self-renewal, and lineage transition from NEP cells to RG. In addition, GRPs were further purified and characterized to study gliogenesis. The results suggested that the early neural lineage development of rESCs in vitro could closely mimic the equivalent process in vivo.

Keywords: rhesus monkey embryonic stem cell (rESC), differentiation, neurogenesis, gliogenesis, neural lineage, notch signaling

Cell Research (2008) 18:s148. doi: 10.1038/cr.2008.238; published online 4 August 2008

Correspondence: Weizhi Ji

E-mail:wji@mail.kiz.ac.cn 